\title{
The reflex sympathetic dystrophy syndrome, associated with traumatic myelopathy ( Pain, 37 (1989) 187-192)
}

\author{
Gary Davidoff \\ Rehabilitation Medicine Service, VAMC, Ann Arbor, MI (U.S.A.), and Dept. of Physical Medicine and Rehabilitation, University of \\ Michigan Hospitals, 1500 East Medical Center Drive, Ann Arbor, MI $48109-0042$ (U.S.A.) \\ (Received and accepted 12 July 1989)
}

\section{Dear Editor,}

Thank you for the opportunity to respond to Professor Churcher's comments concerning the above manuscript. We agree that reflex sympathetic dystrophy syndrome (RSDS) should be considered a subset of the sympathetic mediated pain states (SMPS). However, we strenuously disagree that the reversibility of symptoms with a sympathetic block is a necessary and sufficient condition for confirming the diagnosis. We have seen several patients in our clinic at the University of Michigan, who meet all the criteria for definite RSDS, but have no consistent relief with a properly performed sympathetic block vs. injection with saline. We would suggest that sympathetic blockade, as with 3-phase bone scans, can be used as an adjunct to confirm the diagnosis of RSDS, but does not have adequate positive predictive values, to be the sine qua non of RSDS. 